\title{
Percutaneous Coronary Intervention of Saphenous Vein Graft in Post-CABG Patients- Experience at a Tertiary Cardiac Centre
}

\author{
AHM Waliul Islam¹, Shams Munwar², AQM Reza ${ }^{3}$, Shahabuddin Talukder ${ }^{4}$, Azfar H Bhuiyan ${ }^{5}$, Tamzeed Ahmed ${ }^{6}$, \\ Zulfiqur Haider ${ }^{7}$, Sohail Ahmed ${ }^{8}$
}

\begin{abstract}
:
Background: Percutaneous Coronary Intervention (PCI) of obstructed \& atheromatous venous graft is a real challenge for interventionist to deal with as SVG PCI patients are usually older with significant coronary \& non coronary comorbidities. SVG usually presents a degenerated pattern of atherosclerosis with complex friable thrombosis prone lesions, higher risk of distal embolization, poorer long-term outcome with higher ISR rate
\end{abstract}

Objectives: In the current era, with the advent and availability of different Drug Eluting Stents, PCl of SVG vessel is an alternative to re-do surgery for the occlusion of graft vessel. Although, $\mathrm{PCl}$ is associated with higher risk of instent restenosis, target vessel repeat revascularization, myocardial infarction or death. Uses of embolic protection devices is class I indication by ACC/AHA for SVG PCI. Therefore, we have carried out this prospective study, to see the outcomes of SVG vessel PCl at our center.

Methods and materials: Patients were enrolled in this observational non-randomized prospective cohort, who underwent routine CAG for the post CABG angina, shortness of breath, dyspnea on minimal exertion or hospital admission with MI, NSTEMI, Angina II-III and planned for $\mathrm{PCl}$ of occluded graft vessel. Total 50 patients were enrolled in this study. Distal protection devices were not used in most of the cases as financial costing is an issue.

Results:Total 46 patients were enrolled in this observational study. Average age of the patient population was $(62.1 \pm 10.8)$, female $3(6.5 \%)$ : male $43(93.5 \%)$, BMI
(24.9 \pm 2.9$)$. Among the CAD risk factors; DM 30(60\%), HTN $34(68 \%)$, Dyslipidemia 31(62\%), Smoking 12(6\%) And family history of IHD $9(18 \%)$. Graft vessel occlusion occurred average $(11.5+5.4$ Yrs.) after CABG. SVG to OM is the commonest vessel, that developed significant stenosis in $27(56 \%)$, followed by LAD 9(16.1\%), RCA 6(10.7\%), PDA 6(10.7\%), PLB 3(5.4\%), DG 3(5.4\%)m and LIMA-LAD 1(1.8\%). Total 63 stents were deployed in 56 vessels of 46 patients. Double or overlapping stents were deployed; two stents in $11(17.5 \%)$ and three stents in $1(2 \%)$ vessel. One patient had recurrent ISR of SVG-OM stents and had PCl at our center and elsewhere. Common DES were, Sirolimus 25(39.7\%), Everolimus 22(34.9\%), BMS 9(14.3\%), Zotarolimus $3(4.8 \%)$. Average stent size was $3.3 \mathrm{~mm}$ in Diameter. Total $3(6 \%)$ patient died, in 1 month to $2 y r s$ after the procedure. No acute or late complications were noted in this small group of patients and all were doing well at 12-24 months OPD follow-up.

Conclusion: We found that our patients developed graft vessel occlusion on an average 11yrs, after CABG. OM is the commonest territory to develope significant stenosis. PCI of SVG survival outcome was $93.5 \%$ (43 patient) in this very primitive observational cohort and all were doing well with OPD follow-up. Thus, we recommend percutaneous coronary intervention of occluded or stenosed graft vessel as an alternative to re-do surgery in this part of the world.

Key Words: SVGs, PCI, CABG

1. Consultant, Interventional Cardiology, Evercare Hospital, Dhaka, Bangladesh

2. Senior Consultant, Interventional Cardiology, Evercare Hospital, Dhaka, Bangladesh

3. Senior Consultant, Interventional Cardiology, Evercare Hospital, Dhaka, Bangladesh

4. Senior Consultant, Interventional Cardiology, Evercare Hospital, Dhaka, Bangladesh

5. Specialist, Interventional Cardiology, Evercare Hospital, Dhaka, Bangladesh

6. Senior Consultant, Interventional Cardiology, Evercare Hospital, Dhaka, Bangladesh

7. Senior Consultant, CT Surgery, Evercare Hospital, Dhaka, Bangladesh

8. Senior Consultant, CT Surgery, Evercare Hospital, Dhaka, Bangladesh

DOI: https://doi.org/10.3329/bhj.v35i1.49137

Copyright $\odot 2017$ Bangladesh Cardiac Society. Published by Bangladesh Cardiac Society. This is an Open Access articles published under the Creative Commons Attribution-NonCommercial 4.0 International License (CC BY-NC). This license permits use, distribution and reproduction in any medium, provided the original work is properly cited and is not used for commercial purposes. 


\section{Introduction:}

Saphenous vein grafts (SVGs) are commonly used during coronary artery bypass graft surgery (CABG) for severe coronary artery disease. However, SVGs are prone to both degeneration and occlusion, leading to poor long-term patency and thereby graft occlusion. Rates of SVGs failure in the first $12-18$ months may be as high as $25 \% .^{1-}$ ${ }^{2}$ Percutaneous coronary intervention $(\mathrm{PCl})$ of patients with obstructive atherosclerotic disease in coronary saphenous vein bypass graft (SVG) remains a challenge in interventional cardiology. Usually, this groups of patients are older, suffer significant comorbidities. SVG PCI is associated with worse clinical outcomes compared with native coronary artery $\mathrm{PCl} .{ }^{3}$ Saphenous veins present degenerative pattern of atherosclerotic plaque with complex, friable, thrombotic-prone lesion. Intervention of diseased SVGs carry a higher risk of distal embolization and poorer long-term outcomes. ${ }^{4-5}$ The important reason of poorer outcomes in SVGs PCI is the embolization of atherothrombotic debris into native circulation, often resulting in periprocedural $\mathrm{Ml}$ or reduce antegrade flow.

With the advent of interventional procedures with different drug Eluting stents and distal protection devices, intervention of SVGs in our patient population is an alternative to Re-do CABG as the later carries a risk with different comorbidities. Exact data of $\mathrm{PCl}$ in SVGs in our Bangladeshi patient population not yet available. Therefore, we have carried out this observational study to see the procedural success, morbidity and 1-year survival outcome in this subset of patient population with SVGs stenosis.

\section{Material and Methods:}

Patients were enrolled in this observational nonrandomized prospective cohort, who underwent routine CAG for the post CABG angina, shortness of breath, dyspnea on minimal exertion or hospital admission with Angina II-III and planned for $\mathrm{PCl}$ of occluded graft vessel. Total 50 patient were enrolled in this study. Distal protection devices were not used in most of the cases as financial costing is an issue. Most of the studied patient had their $\mathrm{PCl}$ or $\mathrm{CABG}$ done here or somewhere else and kept on DAPT with Aspirin and Clopidogrel. For those in our center, all Patient were routinely loaded with pre-procedural Clopidogrel 300mg and Aspirin 300mg with post procedural maintenance doses Clopidogrel $150 \mathrm{mg}$ and Aspirin 150mg. Average age of the patient population was $(62.1 \pm 10.8)$, Female $3(6.5 \%)$ : Male $43(93.5 \%)$, BMI (24.9 \pm 2.9$)$. Among the CAD risk factors; DM 30(60\%), HTN 34(68\%), Dyslipidemia 31(62\%), Smoking 12(6\%) And FH 9(18\%).

\section{Results:}

Table1. shows the profile of studied patient. Total 46 patient were enrolled in this observational study. Figure 1. Shows distribution of Female were 3 (6.5\%) and male 43 (93.5\%).
Figure 2. Shows, average LVEF, FBS level, $\mathrm{HbA} 1 \mathrm{C}$ and Creatinine level. Figure 3 shows average level of lipid profile. Figure 4. Shows percentage distribution of CAD risk factors, HTN followed by Dyslipidemia, DM, FH and smoking. In this study, we found that Graft vessel occlusion occurred at an average (11.5+5.4 Yrs.) after CABG. As shown in Figure 5,SVG to OM is the commonest vessel, that developed significant stenosis and found in $27(56 \%)$, followed by SVG to LAD 9(16.1\%), SVG-RCA 6(10.7\%), SVG-PDA 6(10.7\%), SVG-PLB 3(5.4\%), SVG-DG 3(5.4\%) and LIMA-LAD 1(.8\%). Total 63 stents were deployed in 56 vessels of 46 patients. One patient had multiple stents and several times $\mathrm{PCl}$ for recurrence of symptoms and ISR in OM territory. Double or overlapping stents were deployed; two stents in $11(17.5 \%)$ and three stents in 1 (2\%) vessel. Figure 6 shows the percentage distribution of different stents used; common DES were, Sirolimus 25(39.7\%), Everolimus 22(34.9\%), BMS 9(14.3\%), Zotarolimus $3(4.8 \%)$. Average stent size was $3.3 \mathrm{~mm}$ in Diameter. Total $3(6 \%)$ patient died; within 1 month to $2 y r s$ after the procedure. No acute or late complications noted in this small group of patients and doing well at 12-24 months OPD follow-up. Figure 7. Shows the distal protection device and by using it, PCl of SVG graft vessel. Figure 8 shows the distal filter and by using it, $\mathrm{PCl}$ of SVG graft vessel. Figure 9. Shows PCl of SVG-RCA and Relook CAG after 2 months.

Table-I

Demographic Profile of the patients

\begin{tabular}{lc}
\hline Number & (F3/M43) \\
\hline Age $(\mathrm{yrs})$ & $62.1 \pm 10.8$ \\
$\mathrm{BMI}\left(\mathrm{kg} / \mathrm{m}^{2}\right)$ & $24.9 \pm 2.9$ \\
$\mathrm{SBP}(\mathrm{mmHg})$ & $128.0 \pm 19.0$ \\
$\mathrm{DBP}(\mathrm{mmHg})$ & $76.2 \pm 8.3$ \\
No. of CAD Risk Factor & $2.8 \pm 0.9$ \\
\hline
\end{tabular}

\# Data were presented as Mean \pm SD

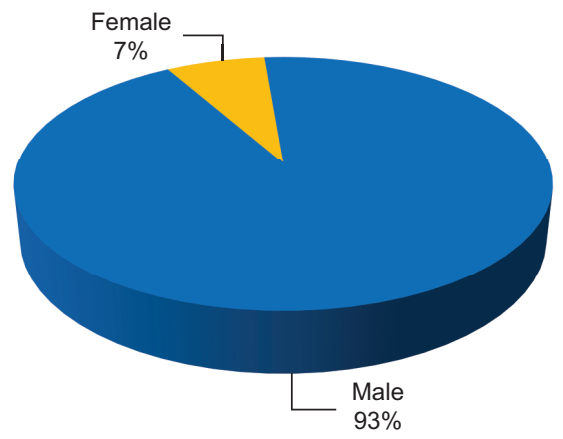

Fig.-1: Percentage distribution of Male and Female in the studied population 


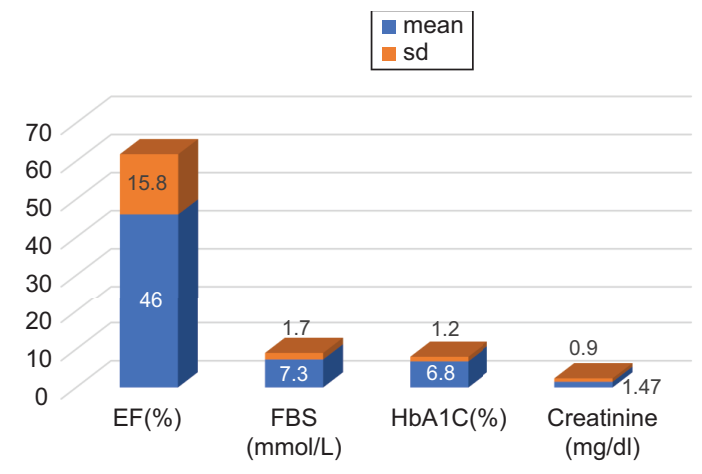

Fig.-2: Shows $L V E F, F B S, H b A 1 C$ and Creatinine level

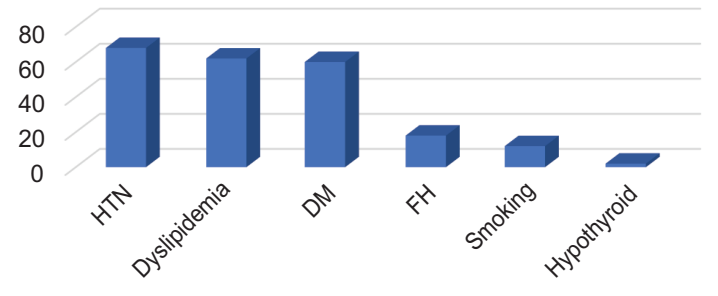

Fig.-4: Percentage distribution of CAD Risk Factors

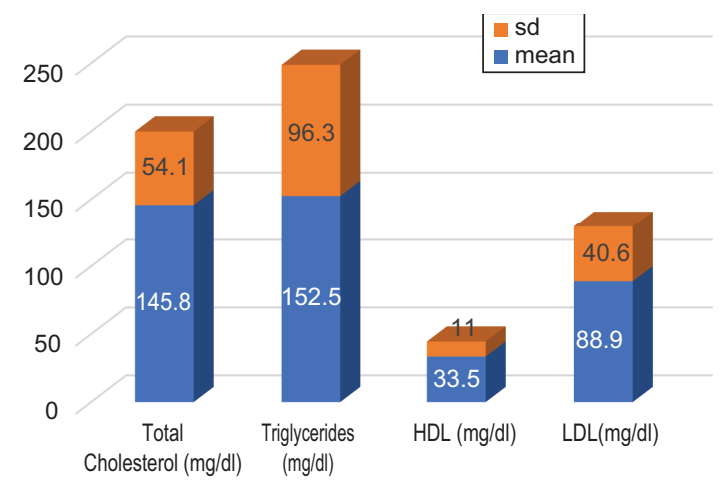

Fig.-3: average shows Lipid Profile

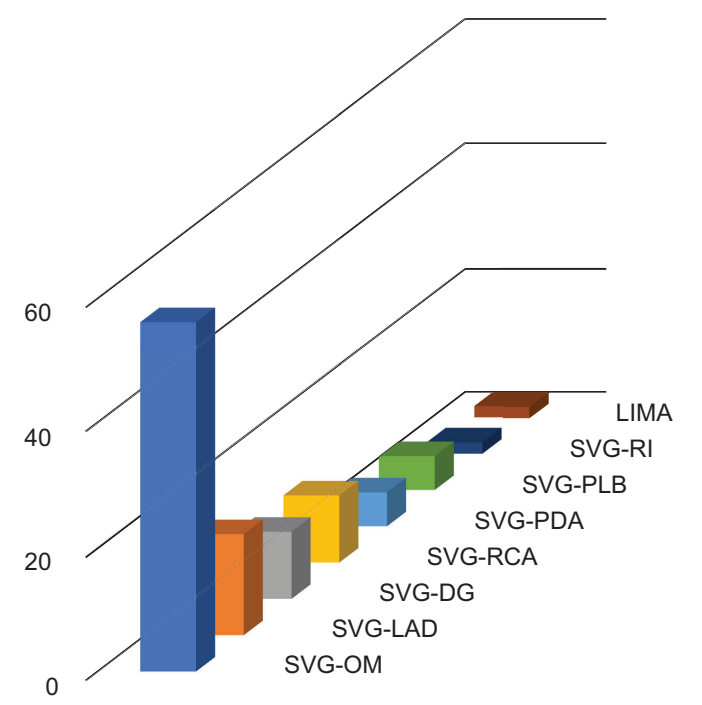

Fig.-5: Stented territory of SVG to Native Coronaries

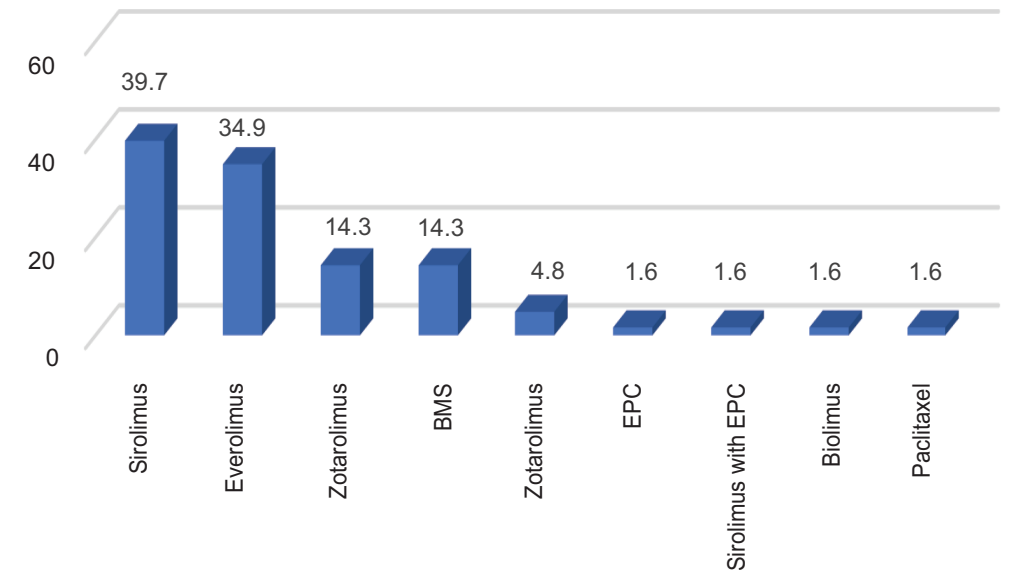

Fig.-6: Distribution of Stents 


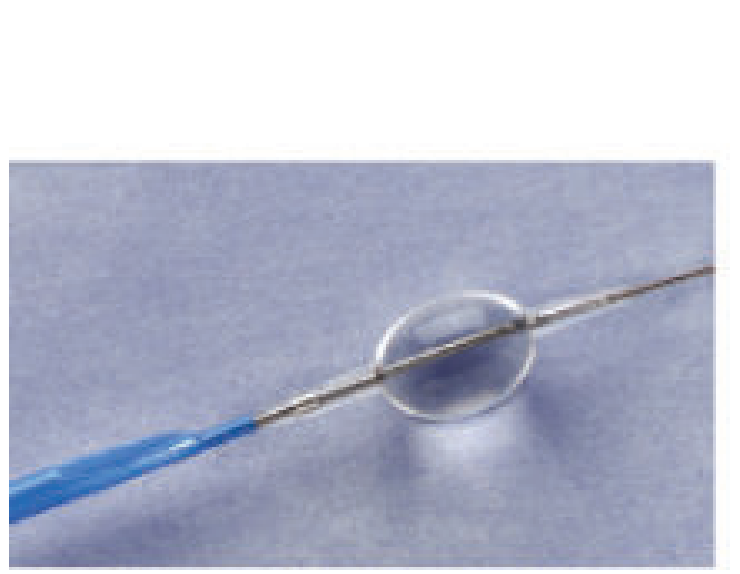

(a)

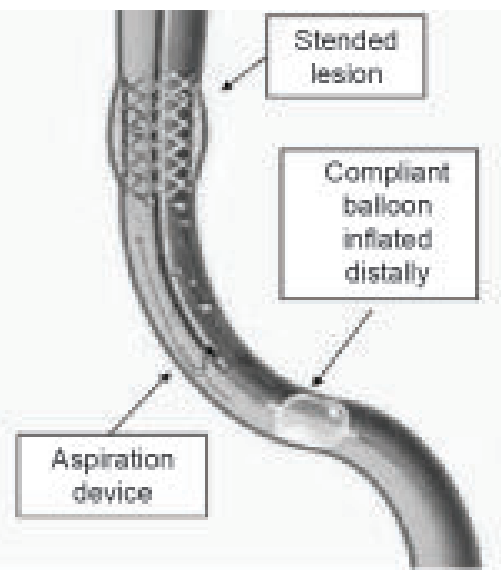

(b)

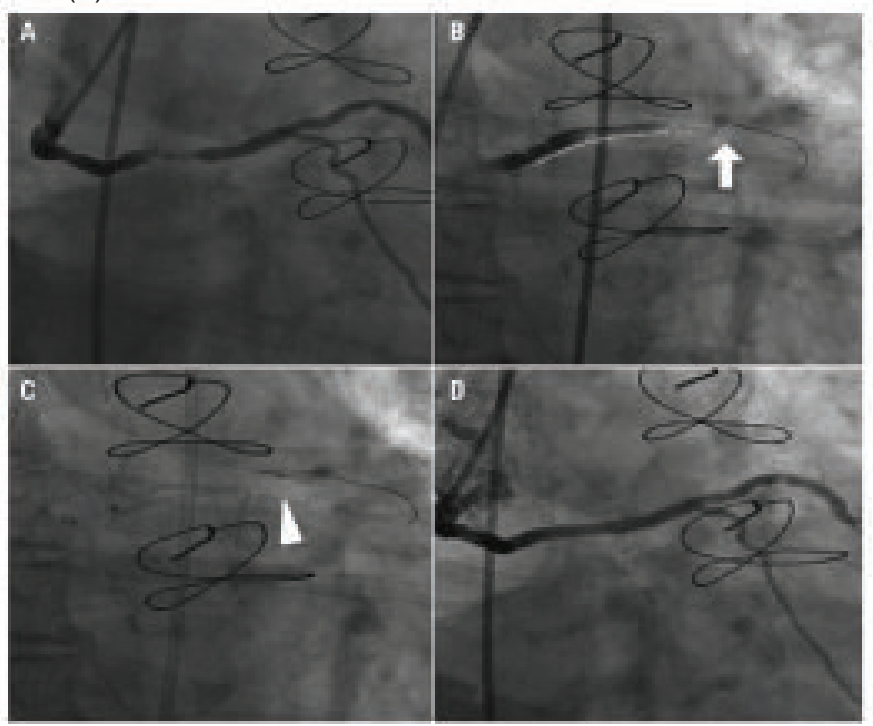

(C)

Fig.-7 a). Distal occlusion device (PercuSurgeGuardWire; Medtronic Inc., Minneapolis, MN, USA). b). Distal occlusion device, graphical representation. c). Distal occlusion device mechanism of action: A) Severe stenosis in the mid portion of a saphenous vein graft to the left circumflex coronary artery. B) Distal occlusion non-compliant balloon inflated (arrow) with a stent positioned at the level of the lesion (dotted line). C) Manual debris aspiration (the arrowhead indicates the tip of the aspiration catheter) still with inflated distal occlusion balloon in place. D) Final result.

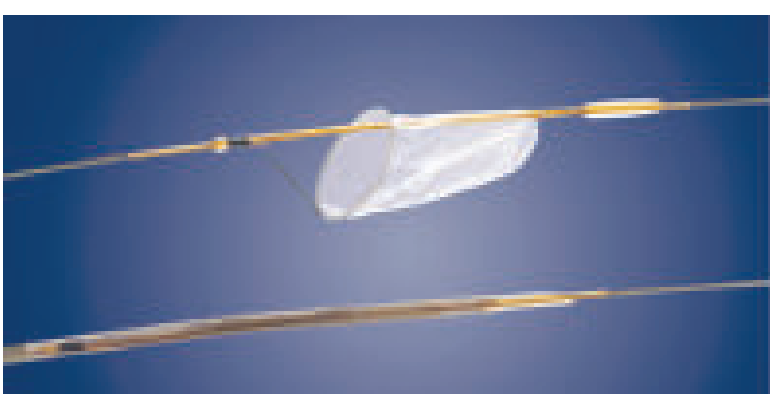

(a)

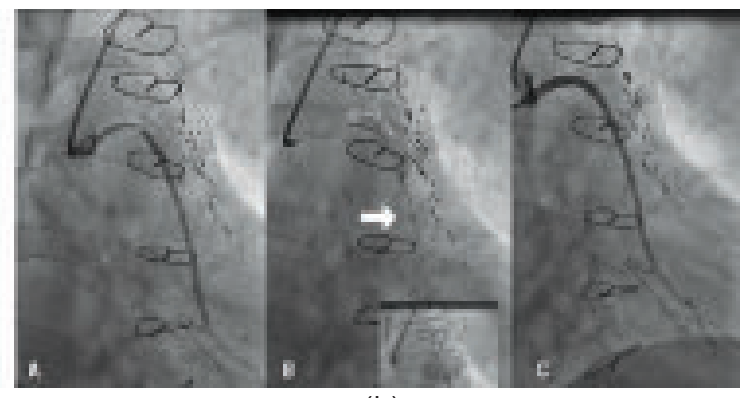

(b)

Fig.-8: a). Distal filter (FilterWire EX®; Boston Scientific, Natick, MA, USA). b). Distal filter mechanism of action: A) long severe proximal stenosis of a saphenous vein graft to the left circumflex coronary artery. B) distal filter (arrow) (Emboshield; Abbott Vascular, Redwood City, CA, USA) and stent in place (dotted line). In the small bottom left quadrant, a second stent is deployed in proximal overlap with the first one, still with the filter in place. C) Final result. 


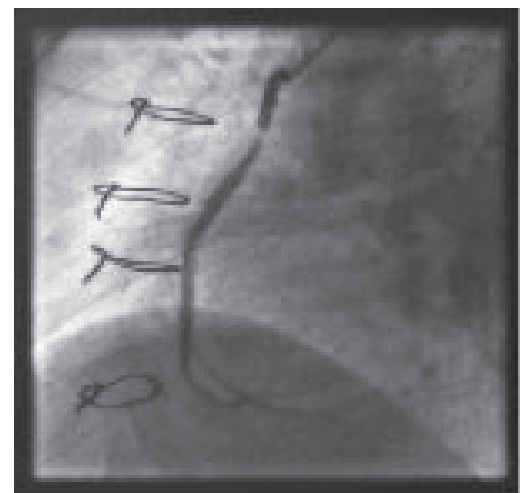

(a)

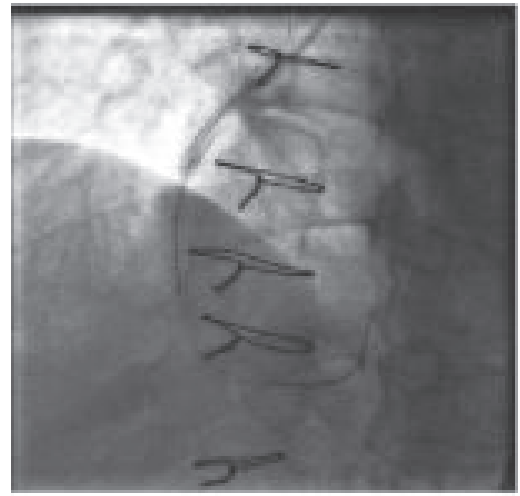

(d)

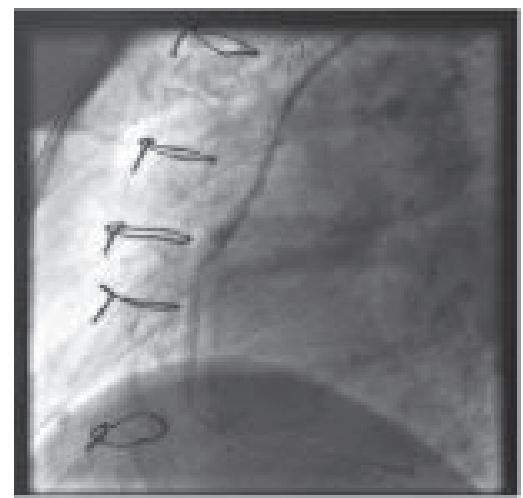

(b)

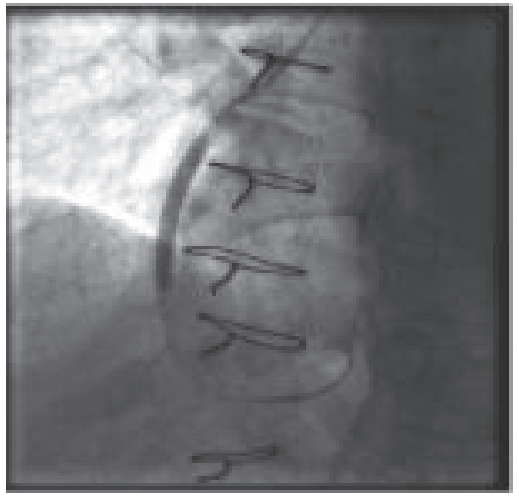

(e)

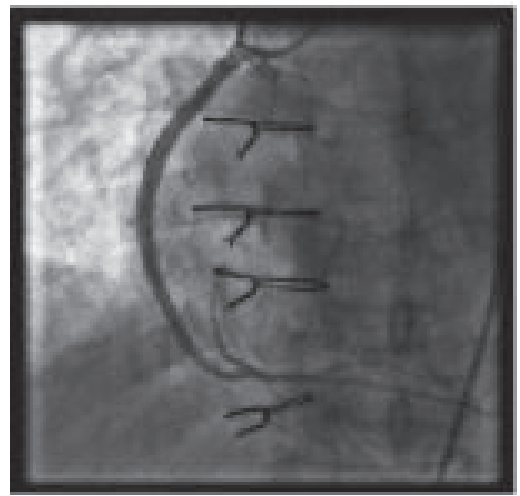

(g)

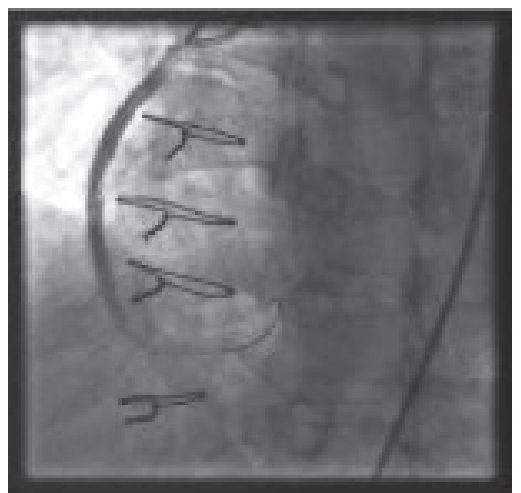

(c)

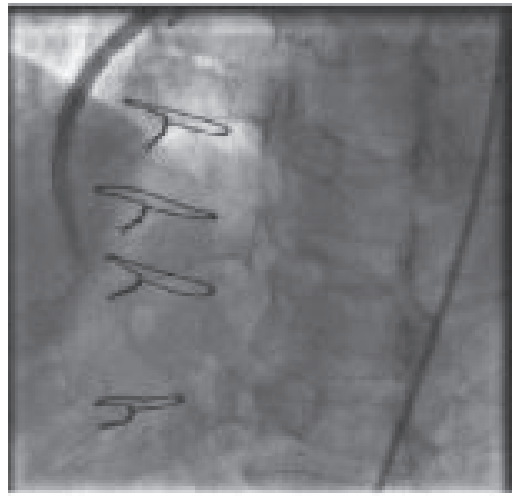

(f)

Fig.-9: a). Acute Inf MI, 16 years after CABG, Thrombolysed, Post MI Angina SVG PCI to RCA. $b$ and c). After directly stenting the plaque, initially looked ok. $2^{\text {nd }}$ injection showing new thrombus/ degenerated materials after the stent. $d$, e,f) $A 2^{\text {nd }}$ stent deployed directly followed by no flow and slow flow. After Adenosine, Nitrates, Integrillin, some flow returned with native artery visualization and improvement of symptoms and hemodynamics g). Relook CAG 2 months later showed patent SVG-RCA stent with no residual stenosis

\section{Discussion:}

Saphenous vein graft (SVGs) are still commonly used for surgical revascularization of coronary artery although are related to poor long-term patency. ${ }^{6}$ Full arterial revascularization in coronary artery bypass graft (CABG) procedure, despite related improved clinical outcomes, is still seldom achieved. ${ }^{7}$ For this reasons , percutaneous coronary intervention of SVGs is being routinely done in daily practice, accounting approximately $6-10 \%$ of total $\mathrm{PCl}$ volume with optimal clinical outcome compare to 
native coronary $\mathrm{PCl}$ with higher rate of in-stent restenosis (ISR), target vessel revascularization(TVR)m, death, MI. ${ }^{8}$ In this scenario, DES vs BMS in SVGs have shown favorable outcome in DES than compare to BMS regarding angiographic and clinical short and midterm restenosis at follow-up. ${ }^{9-10}$

Although, advancement of interventional cardiology with newer technology along with the availability of hard wires for SVGs intervention, the optimal satisfactory outcome doing $\mathrm{PCl}$ in SVGs remain unsatisfactory. Several study on SVGs PCl either with First Generation DES vs BMS has shown different outcomes. For instances in in SAVED (saphenous vein de NOVO) trial reported that compared with balloon angioplasty, BMS were associated with higher peri-procedural success. DIVA trial, ${ }^{11-12}$ where $88 \%$ DES stents were of Secondgeneration DES, at 1-year follow-up the incidence of target vessel failure (Primary endpoint of composite of cardiac death, target vessel MI or TVR) was not different compare to BMS. Moreover, at median 2.7 years follow up no significant difference between the group in terms of all cause death, MI, stent thrombosis, stroke, bleeding or other secondary outcomes were observed. In the ISARCABG trial ${ }^{13}$ randomly assigned 610 patients to first generation DES or BMS, and reported lower 12-month incidence of target vessel revascularization in the DES group ( $7 \%$ vs $13 \%, \mathrm{P}=0.01$ ) and no significant differences in all-cause mortality, myocardial infarction and definite or probable stent thrombosis as com[pared with BMS. BASKET- SAVAGE trial ${ }^{14}$ has lower incidence of target vessel revascularization in the DES group (4.5\% vs $19.1 \%$ at 3 years $p<0.001$ ) and lower rate of MACE in DES group ( $30 \%$ vs $12 \%$, HR 0.33, $\mathrm{P}=0.001$ ). In RRISC (Reduction of Restenosis in Saphenous vein grafts with Cypher), ISR rate at six months significantly reduced in Cypher, consistently with a drop of TLR and TVR. ${ }^{6}$ Similarly, SOS (Stent of saphenous Vein Grafts) trial, paclitaxel eluting stent showed lower rate of ISR in paclitaxel with significant reduction of TLR and TVR in Taxus arm than BMS. Thus, both RRISC and SOS uses first generation DES with significant advantages of drug eluting stents in treating de-novo SVG.

Percutaneous coronary intervention of saphenous vein graft lesion is associated with a uniquely high-risk periprocedural myocardial infarction. (MI) and mortality-much higher than routine native coronary. Distal embolization manifested as slow-flow and no-reflow (SNFR) in 10$15 \%$ of SVG PCls. SVG plaques are large, soft, friable lipid rich, containing large necrotic debris, cholesterol crystals and foam cell, and are often associated with overlying thrombus. During $\mathrm{PCl}$ of SVGs, distal embolization of this particle may lead to platelet and leukocyte activation, release of vasospastic mediators (serotonin, endothelin). And activation of chemotactic mediators (tissue factor, thrombin/anti-thrombin III complex and prothrombin fragments. Thus, lead to triad of microvascular embolization, spasm, and thrombosis manifesting as SFNR. ${ }^{15}$

Uses of Embolic protection devices is a class I indication according to the ACC/AHA/SCAI PCl guideline ${ }^{16}$ when feasible, to decrease the risk of distal embolization, noreflow, and periprocedural myocardial infarction. This recommendation was based on a single randomized controlled trial, the SAFER study, which showed significant reduction in major adverse cardiac events (MACE) with the use of a distal balloon occlusion device. ${ }^{17}$

Bangladesh has emerged as one of the leading countries, in this part of world where most of the big city, cardiac catheterization laboratory is available. For last decades, we set to world class standard in treating all types of coronary intervention, both elective and primary. Doing all normal to complex, including lifesaving intervention with the support of IABP when indicated.

$\mathrm{CABG}$ is the choice of revascularization intreating multivessel disease, where $\mathrm{PCl}$ is very expensive as we don't have individual medical insurances. Most of our patient are physically not fit enough to proceed for Re-do surgery. Moreover, intervention of SVGs PCI single or multiple, interventionist needs to be expert enough to treat such de-novo SVG. Till now, we don't have exact clinical scenario or data of survival outcome follow-up, in this subset of cardiac patient.

To our knowledge, for the first time, we have carried out this observational non-randomized prospective study in our center. Patient with significant SVGs occlusion with ongoing Angina or dyspnea on effort or at rest, underwent for $\mathrm{PCl}$ were studied. We found that SVGs to OM are prone to develop occlusion possibly due to anatomic location of OM is of the predisposing factor. Also, stents in SVG to OM is susceptible to develop recurrent ISR. One of our patients had several times stenting in SVG to OM for recurrent ISR. Thus, PCI to SVG to OM may not be suitable or recommended until it is deemed necessitate to relief ongoing angina or the OM territory is big enough with viable myocardium.

The long-term success of surgical coronary revascularization is limited by accelerated atherosclerosis and intimal fibrosis of saphenous veins after its use as a vascular conduit. 
At 1-year incidence in 1 or more total SVG occlusion has been reported to be high as $41 \%$ after on-pump by-pass surgery. ${ }^{18-19}$ Because of increased morbidity and mortality with repeat coronary artery bypass graft surgery, SVG intervention is considered by many to be the preferred revascularization in patients with diseased SVGs and accounts for approximately 5 to $10 \%$ of all percutaneous coronary intervention. ${ }^{20-21}$

\section{Conclusion:}

Saphenous vein grafts (SVGs) are commonly used conduits for surgical revascularization of coronary arteries but are associated with poor mid and long-term patency rates. We know that $\mathrm{PCl}$ of SVGs is associated with worse clinical outcomes including higher rates of in-stent restenosis, target vessel revascularization, myocardial infarction and death compared to $\mathrm{PCl}$ of native coronaries. The higher rate of MI with SVG PCl compared with nonSVG PCI supports the role of thrombosis in the pathogenesis of SVG failure. However, reduced flow rate, altered shear stress, pronounced neointimal proliferation and differential plaque and vessel (absence of media) structure and composition are also likely to contribute SVG failure. ${ }^{22-23}$ Although, longer term DAPT might improve the better survival outcome, the chances of bleeding risk among this group of patients who are mostly older ages. Rather, intensified DAPT may be beneficial in this group of patients. ${ }^{24} \mathrm{Re}$-do CABG is always complex, carries a higher risk of morbidity and mortality. Thus, we may conclude that in our context, PCI to SVGs may be the choice of revascularization.

\section{Future perspectives:}

This is very primitive observational study of SVG PCI of post CABG patient admitted for coronary angiogram for ongoing chest pain or exertional dyspnea. One of the major drawbacks of our Bangladeshi patient population are not follow-up with the same physician or hospital. Noncompliance to medication and lifestyle modification along with control of CAD risk factors harmful effects are also reason to recurrence of symptoms with hospitalization and revascularization. We know that, SVGs are prone to degeneration and occlusion, leading to poor long-term patency and thereby graft occlusion. We found recurrence of ISR in graft vessel is not an uncommon phenomenon. Thereby, needs multicenter involvement to develop common consensus in treating this group of Graft occlusion patient. Thus, to treat early and reduce repeat re-hospitalization and target vessel revascularization.

\section{References:}

1. Fitzgibbon GM, Kafka HP et al. Coronary bypass graft fate and patient outcome: angiographic follow up of 5065 grafts related to survival; and reoperation in 1388 patients for 25 years. J Am Coll Cardiol 1996:25:616-26

2. Cataldo G, Braga M,Pirotta $\mathrm{N}$ et al. Factors influencing 1-year patency of coronary artery saphenous vein grafts. Circulation. 1993:88: 1193-8

3. Bundhoo SS, Kalla $\mathrm{M}$ et al. outcomes following PCI I patients with previous CABG: a multicenter experience. Catheter Cardiovasc Interv 2011;78: 169-76

4. Fireman $\mathrm{A}$, Rechavia $\mathrm{E}$, Eigkler $\mathrm{N}$ et al Long-term follow-up of a high-risk cohort after stent implantation in saphenous vein grafts. J Am Coll Cardiol 1997; 30:1277-83

5. Pucelikova T, Mehran R, Kirtane AJ et al. Short and long-term outcomes after stent-assisted percutaneous treatment of saphenous vein graft in the drug-eluting stent era. Am J Cardiol. 2008:101:63-8

6. Goldman S, Zadina K, Moritz T et al. Long-term patency of saphenous vein and left internal mammary artery grafts after coronary artery bypass surgery>: results from veterans' affair cooperative study. J Am Coll Cardiol 2004; 44:2149-56

7. Muneretto C, Bisleri G, Negri A et al. Total arterial revascularization with composite grafts improves results of coronary surgery I elderly: a prospective randomized comparison with conventional coronary artery bypass surgery. Circulation 2003:148-suppl 1: 1129-33

8. Brilakis ES, Wang TY, Rao SV et al. Frequency and predictors of drug-eluting stent use in saphenous vein bypass graft percutaneous coronary interventions. JACC Intv. 2010;3: 1068-73

9. Vermeersch P, Agostoni P, Verheye $S$ et al. Randomized double-blind comparison of Sirolimus Eluting stent versus bare metal stent implantation in diseased saphenous vein graft: six-month angiographic, intravascular ultrasound and clinical follow-up of the RRISC trial. J Am Coll Cardiol 2006:48:2423-31

10. Brilakis ES, Lichtenwalter C, Abdel Karim AR et al. Continued benefit of paclitaxel eluting stent versus 
bare metal stents implantation in saphenous vein graft lesion during long-term follow-up of SOS (stenting of Saphenous Vein Graft) trial. JACC Cardiovasc Interv. 2011; 4:176-82

11. Brilakis ES, Edson R, Bhatt DL et al. Drug Eluting Stents versus bare metal stents in saphenous vein grafts: a double-blind randomized trial. Lancet 2018:391:1997-2007

12. Brilakis ES, Edson R, Bhatt DL et al. Rationale and design of the drug eluting stents vs bare metal stents in saphenous vein graft angioplasty (DIVA) trial. Clin Cardiol 2017; 40:946-54 Lancet 2018:391:1997-2007

13. Mehili J, Pache J, Abdel -Wahab M et al. Drug eluting vs bare metal stents in saphenous vein graft lesions (ISAR-CABG): a randomized controlled superiority trial. Lancet 2011; 378:1071-78

14. Jeger R. Study to test the efficacy and safety of drug eluting vs bare metal stents for saphenous vein graft interventions (BASKET-SAVAGE), European Society of Cardiology metting Rome, Itakly. Aug 27$31,2016.5025$

15. Sharma S, Lardizabal JA Singh $S$ et al. Intra-graft abximab and verapamil combined with direct stenting is a safe and effective strategy to prevent slow-flow and no-reflow phenomenon in saphenous vein graft lesions not associated with thrombus. Recent Pat Cardiovasc Drug Disc 2012; 7:152-159

16. Levine GN, Bates ER, Blankenship JC et al. 2011 ACC/AHA /SCAI Guideline for percutaneous coronary intervention: a report of the American College of Cardiology Foundation/ American Heart Assoc Task Force on Practice Guideline and the society for Cardiovascular Angioghraphy and Interventions. Circulation. 2011:124: e574-e651
17. Widimsky P, Straka Z, Stros $\mathrm{P}$ et al. One-year coronary bypass graft patency: a randomized comparison between off-pump and on-pump surgery angiographic results of the PRAGUE-4 trial. Circulation 2004;110: 3418-23

18. Halabi AR, Alexander JH, Shaw LK et al. Relation of early saphenous vein graft failure to outcomes following coronary artery bypass surgery. Am J cardiol 205;96: 1254-9

19. Brodie BR, Wilson H, Stuckey $\mathrm{T}$ et al. Outcomes with drug eluting versus bare-metal stents in saphenous vein graft intervention: results from the STENT group. JACC Intv 2009; 2:1105-12

20. Brilakis ES, Rao SV, Banarjee S et al. Percutaneous coronary interrogation in native arteries versus bypass grafts in prior CABG patients: a report from national cardiovascular data registry. JACC Cardiovasc Intervention 2011:4:844-50

21. Morrison DA, Sethi G, Sacks J et alPercu8taneous coronary intervention versus repeat bypass surgery for patients with medically refractory myocardial ischemia: AWESOME randomized trial and registry experience with post CABG patient. Am J cardiol 2002; 40:1951-4

22. Bulkley $B H$, Hutchins $G M$ et al. Accelerated "atherosclerosis". A morphologic study of 97 saphenous vein coronary artery by-pass grafts. Circulation 1977; 55:163-169

23. Lichtenwalter $\mathrm{C}$, de Lemos JA, Roesle $\mathrm{M}$ et al. Clinical presentation and angiographic characteristics of saphenous vein graft failure after stenting: insights from the SOS (stenting of saphenous vein graft) trial. JACC Cardiovasc Interv. 2009;2":855-860

24. Redfors B, Genereux P, Witzenbicler B et al. Percutaneous Coronary Intervention of Saphenous Vein Graft. Cir Cardiovasc Interv 2017. 10: e4953 